\title{
Tissue Scroll
}

National Cancer Institute

\section{Source}

National Cancer Institute. Tissue Scroll. NCI Thesaurus. Code C99276.

A piece of tissue cut on a microtome so that it rolls up on itself, giving it an appearance similar to a paper scroll. 